\title{
El Enfoque Comunicacional en la formación de Terapeutas Ocupacionales: Aspectos teóricos y propuestas de aplicación
}

\author{
Rueda C.,Laura(1); Rueda C.,J orge(2) \\ (1)Terapeuta Ocupacional. Magíster en Bioética. Prof. Asistente. U. de Chile. Escuela \\ de Terapia Ocupacional.(2)Académico Depto. Lingüística y Literatura, Facultad de \\ Humanidades Prof. Asociado. U. Santiago de Chile \\ Contacto > > email: Irueda@med.uchile.cl Fono 9786344 email: \\ jrueda@lauca.usach.cl \\ Referencia > > Rueda C., Laura; Rueda C., J orge. "El Enfoque Comunicacional en la \\ formación de Terapeutas Ocupacionales: Aspectos teóricos y propuestas de \\ aplicación. "Revista Chilena de Terapia Ocupacional. №5. Noviembre 2005.
}

\section{- Abstract}

Based on the functional communicative model, a proposal to be applied on the teaching of health professionals is here presented. This approach favors the communicative process as a mediation principle of the human practice, and overcomes the limitations that the conventional educational lineaments, among other matters, have demonstrated to possess.

First, and analytic view of the "communicative approach" is exposed, highlighting the important role it has had in the revision of certain cultural paradigms. Second, the basic principles are projected so as to support and complement the complexity of the knowledge that needs to be systematized in the training of occupational therapists.

\section{Resumen}

Este ensayo presenta una aproximación integral, basada en el desarrollo de un enfoque comunicativo funcional, para los procesos formativos de los profesionales de la salud y la educación. En primer lugar, se expone una visión sistemática y analítica de la perspectiva comunicacional para ser aplicada como contexto a las situaciones formativas. En segundo lugar, se proyecta la utilización de este enfoque integral para situar la complejidad de saberes que deben sistematizarse en la formación de Terapeutas Ocupacionales.

El enfoque integral-comunicacional es utilizado para identificar y superar las limitaciones de las estrategias de la política educacional convencional, así como, al mismo tiempo ofrece una aproximación alternativa a los principios de una praxis docente más madura.

\section{I ntroducción}

La realidad humana y la diversidad de relaciones que de ella se desprenden dan actualmente relieve, en todas sus manifestaciones, al proceso comunicativo en cuanto principio mediador, simbólico y material. En su interior se descubren las estructuras más profundas del sentido cultural en acción y se posibilita la representación de la realidad social que una colectividad desea construir. Sin ir más lejos, Chile vive desde ya algunos años una reforma en los niveles de educación básica y media, cuya matriz curricular dice relación 
con la necesidad de que la actividad educativa sustente los procesos, conocimientos y destrezas que le dan marco, sobre la base del desarrollo de estrategias metacognoscitivas ${ }^{(i)}$ producto del enfoque comunicacional funcional. Esto, en otras palabras, significa que el énfasis en los procedimientos practicados por el docente, respecto del tratamiento de un conocimiento formal por enseñar, debe promover la integración de los estudiantes al proceso educativo mediante el discurso / habla-lengua ${ }^{\text {(ii) }}$ como instrumento esencial de comunicación. Parece una obviedad, pero la realidad educativa dice otra cosa.

Relacionar la enseñanza como una labor fundamentada en la constante comunicación, es decir, como acto que aspira a enseñar a actuar lingüísticamente de manera satisfactoria y, sobre todo, en conciliación con un contexto social determinado implica una revisión general de la orientación de todo un sistema que, por tradición, se ha encapsulado casi sólo en la transferencia conceptual del conocimiento. Sin duda que esto último es una condición necesaria y deseable en toda actividad humana y, más aún, profesional. No obstante, la consideración de una "educación comunicativa" configura una nueva perspectiva desde "donde mirar" las fronteras de paradigmas culturales. En la base de la nueva actuación docente se ubica el desarrollo cognoscitivo: pensar, imaginar, fomentar sensibilidad acerca del entorno cultural y material, participar crítica y constructivamente de la estructura social.

Tomando en consideración lo anterior, toda actividad humana debería estar sustentada en el reconocimiento del otro a través de - básicamente - una orientación comunicativa que permita desarrollar tal competencia $\mathrm{y}$, como consecuencia, promover un "saber actuar" convenientemente para satisfacer las necesidades sociales. Cabe hacerse algunas interrogaciones: ¿Cuánta atención ha recibido el enfoque comunicativo en los distintos programas de formación profesional? Por razones evidentes, parece haber sido más habitual en la enseñanza e investigación lingüísticas, pero ¿qué otros campos disciplinarios han acogido elementos de este modelo para responder al proceso de profundos cambios y refocalizaciones socio-culturales que experimenta América Latina y, en particular, Chile? ¿Cómo contribuir de manera determinante, en cuanto institución educativa, a que el trabajo de los docentes perfeccione una mejor aplicación de la competencia comunicativa que, en el marco social de una "cultura comunicativa" del nuevo siglo, lleve, finalmente, a una cultura de experiencia democrática(iii).

En lo que sigue, se realizará una reflexión acerca de los alcances teóricos y metodológicos del enfoque comunicativo, centrando la atención en su referente clave: la competencia comunicativa. Posteriormente, se hará una intento por aplicar las nociones delimitadas en la tarea anterior al campo específico de la formación inicial del terapeuta ocupacional, con especial dedicación en los roles que al interior del marco acotado le corresponde al docente.

(i) Por estrategias de la metacognición se entienden aquellos mecanismos relacionados con la forma cómo el sujeto puede alcanzar el conocimiento de sus propios procesos mentales. Como consecuencia, el sujeto puede autocontrolar sus propias dinámicas para conocer un fenómeno

(ii) En términos simples, se define al discurso como una cadena hablada. En efecto, todo discurso es fundamentalmente habla, cuya lógica primordial es la contrapartida de la lengua, aún cuando no se le puede disociar de ella. El discurso (habla) es la lengua puesta en movimiento, es su uso; es acontecimiento y no estructura (Cfr. Ducrot y Todorov, 1991:143 y ss.). Se debe sí reconocer que desde la perspectiva sociológica no basta la determinación por separado de lo uno, ni de lo otro (cfr. Ricoeur, 1987: 245 y ss.).

(iii) Es evidente el intento que hoy se observa de parte de muchos sectores sociales por saltar las que hace un tiempo resultaron ser fronteras insuperables, no sólo en cuanto a teorías y metodologías del pensamiento humano, sino también en cuanto a dimensiones ideológicas y paradigmas de la cultura. Basta reconocer en Chile el énfasis dado en los últimos años a la cuestión indígena, a la problemática de género, a la integración de los discapacitados y, en general, a los grupos humanos tradicionalmente marginados. 


\section{" El Enfoque Comunicativo como Metodología .}

El enfoque comunicativo busca desarrollar, como propósito central, la competencia comunicativa; ésta consiste en el conocimiento y el manejo de las "diversas estructuras (...) adecuadas a la intención, tema, destinatarios y contexto en el que el uso comunicativo se produce" (Lomas et al., 1994:23). En palabras más amplias, tal conceptualización abarca el conocimiento del sistema lingüístico y de otros sistemas de signos no verbales, las condiciones en que se usan estos sistemas en atención a la planificación y a la formalización que exigen, y en atención a los contextos y situaciones de comunicación en los cuales son pertinentes.

En este sentido, el enfoque comunicativo que promueve la autonomía en una práctica docente de negociación ${ }^{(\text {iv })}$ y que, como fin, desarrolle la responsabilidad social, implica la consideración de una "cultura comunicativa": la competencia individual se realiza en un ámbito social propicio a la comunicación, donde naturalmente funciona en beneficio de todos los actores. En tal descripción, el sujeto será miembro de un colectivo ( sociedad, grupo profesional, institución educativa, familia, etc.) comunicativo, donde la comunicación como razón de ser fluya, sea dinámica y, fundamentalmente, auténtica. Competencia y cultura comunicativas, como se observa, corresponden a una unidad nuclear que no constituye un fin en sí misma, sino como una etapa del proceso de consolidación de la cultura comunicativa y de conformación de la cultura democrática.

Con el objeto de profundizar en los elementos componentes de la competencia comunicativa, que serán la base del modelo que se ofrecerá para su aplicación a la formación inicial del terapeuta ocupacional, se describirán los principios que los rigen ${ }^{(v)}$. Varias son las categorías o subcompetencias que la constituyen; importa entenderlas como condiciones para la planificación y formalización de programas facilitadores de la orientación de dinámica humana que se viene acotando.

a) Competencia lingüística. Se manifiesta en la habilidad para producir expresiones significativas formadas de acuerdo con las reglas de la lengua, en función de y respetando su significado convencional.

b) Competencia paralingüística. Conocida igualmente como "competencia de la actuación", corresponde a la capacidad de acompañar o sustituir los mensajes verbales con acciones corporales. Se subdivide en kinésica (signos "gestuales, como señas, mímicas, movimientos del cuerpo, etc.) y proxémica (capacidad para modificar las distancias interpersonales y las posiciones espaciales según la naturaleza del acto comunicativo).

c) Competencia pragmática o performativa.. Equivale a la capacidad de integrar las competencias anteriores para lograr un acto comunicativo adecuado a la situación y a la propia intención del hablante, es decir, acorde con los fines deseados por éste. De esta competencia depende la producción de actos comunicativos exitosos (realización de promesas, solicitudes, órdenes, etc.).

d) Competencia discursiva. Permite vincular e integrar las frases y enunciados en el contexto lingüístico pertinente e interactuar eficazmente en discusiones, narraciones, formulación de preguntas, conversaciones, etc.

e) Competencia sociocultural. Capacidad de reconocer las situaciones sociales y las relaciones de roles. Esta competencia posibilita concebir significados propios de una cultura determinada y conocer los rasgos que caracterizan a cada elemento de esa cultura, aplicando, para ello, las categorías propias de esa cultura específica.

f) Competencia social. Es menos lingüística y se vincula con la personalidad del usuario: el deseo de interactuar comprende empatías y la capacidad de manejar situaciones sociales. 
g) Competencia estratégica. Se refiere a los recursos utilizados para mejorar la comunicación o para compensar las fallas surgidas en ella, debido a factores limitantes en la comunicación (por ejemplo, "ruidos") o la incompetencia en alguno de los factores anteriores por parte de los participantes.

h) Competencia intercultural. Identifica la habilidad de una persona para actuar de manera adecuada y flexible al enfrentarse con acciones, actitudes y expectativas de personas de otras culturas. Al mismo tiempo incluye la capacidad de estabilizar la propia identidad en el proceso de mediación entre culturas y la de ayudar a otras personas a estabilizar la suya.

En síntesis, la noción de "competencia comunicativa" se constituye en un importante constructo teórico que sirve de base para aplicar sus componentes en el momento de elaborar programas, preparar y entrenar profesionales de variadas áreas. En lo que sigue, se intenta una aplicación.

(iv) En general, entiéndase por "negociación" el procedimiento humano en el cual los acuerdos finales de una acción se dan en un contexto de participación, consciente y total.

(v) Sin duda, la intención que prima es ofrecer una apretada y esquemática síntesis de la amplia gama de información sobre los aspectos tratados. Se reconoce, sin embargo, la abundante existencia de estudios y referencias bibliográficas a la cual es fundamental remitirse para ampliar el universo conceptual sobre el tema. Por ejemplo, véase- entre otros - a Austin (1982), Berutto (1979), Habermas (1987), Llobera, et al. (1995), Marcos et al. (1991), Parret (1993), Reyes (1990), Searle (1990).

\section{- Articulación de un programa "}

La ocupación del ser humano, como eje central de un proyecto educativo destinado a formar terapeutas ocupacionales en el marco de la teoría comunicacional expuesta; parte desde la premisa que la actividad ocupacional da como resultado un complejo mundo de vinculaciones. En otras palabras, se focaliza en la constante dinámica que envuelve a la condición humana, en el contexto de "hacer" y "hacerse" día a día.

La ocupación desde esta mirada, cumple con el principio de mediación al constituirse como alternativa de síntesis de interacciones que posibilitan acceder al núcleo de los problemas de la salud humana. La comunicación, expresada en la ocupación o actividad humana, permite hallar formas de trascender los círculos viciosos trasformándoles en virtuosos y los ciclos mortales en vitales.

Los conflictos entorno a la salud, requieren de una interacción comunicativa para lograr recuperar un equilibrio beneficioso. El acto de la comunicación tiene lugar cuando el ser humano entra en contacto con otro ser humano, ve en su semejante a su igual. Ello constituye el eje central de la intervención terapéutica generada entre la persona asistida, con alteraciones en la comunicación (círculos vicioso), incomunicación o bloqueos vinculares permanentes (ciclos mortales). Esto es en términos clínicos, enfermedades en la esfera física o mental; en términos sociales las radicales diferencias entre los seres humanos como la discriminación y la incapacidad de reconciliarse.

En este marco, la ocupación cumple también con el principio simbólico porque se aproxima a una actividad valorativa. La interacción comunicativa del hacer humano acerca axiológicamente a las personas pues, fundamentalmente es en ésta red de interacciones básicas, donde se determina las posibilidades evolutivas, el destino y las diversas formas de razonamientos, de los seres humanos; sea en lo colectivo o en lo individual. Ejemplos de este tipo de interacción es el cuidado y la protección del otro y en especial del otro cuando se encuentra en una situación de vulnerabilidad.

La tarea de agrupar diferentes saberes (mucho de ellos venido de diferentes ciencias, como las biomédicas, las humanísticas ) con el interés de formular y desarrollar una ciencia y una disciplina centrada en la 
ocupación merece consideraciones, y sobre todo una fundamentación en base a la reflexión de un ejercicio profesional establecido durante un largo periodo de evolución.

El abordaje del proyecto formativo, del terapeuta ocupacional, requiere desarrollar las competencias comunicativas para favorecer la interacción de un profesional en el contexto que se encuentre, su herramienta es el rediseño de las ocupaciones que el individuo realice. En esta tarea, requiere competencias que le permitan comparar diferentes perspectivas con las demás. Aunque elijamos una interpretación de la condición humana, los demás puntos de vista también pueden ser considerados, lo que aumenta la flexibilidad en la interpretación y evaluación de las diferentes alternativas.

Un programa formativo de este profesional, exige incluir todos los niveles competitivos contando con más de una perspectiva sin rupturas externas ni internas entre ellas, sino como un sistema perfecto y vitalmente interactivo.

Examinar el desarrollo de las bases conceptuales, fundamentos y aplicaciones prácticas de la ciencia de la ocupación, a través del curriculum formativo del profesional terapeuta ocupacional y especialista en ciencia de la ocupación, es una tarea que sigue 4 senderos, que se van desarrollando integralmente:

1. Desde la malla curricular actual, que ofrece el Plan de Estudio de la Carrera, enmarcado en la programación del centro matriz. Es decir, desde las asignaturas repartidas en los 5 años de formación.

2. Desde una visión integral y transversal del curriculum. Considerando, los saberes centrales de la ciencia de la ocupación que se van generando a lo largo del Plan.

Desde el interés propio de formación de un especialista en Ciencia de la Ocupación. >Desde el interés propio de formación de un profesional Terapeuta Ocupacional.

Una breve revisión de los elementos, que van generándose en cada una de estas vías de análisis, podemos exponerlos a continuación:

\section{Desde la malla curricular actual.}

a) Asignaturas Básicas:

Aportan el conocimiento de la estructura, función y crecimiento del ser humano, tanto físico como psíquico en su relación con el ambiente. Además, se aportan, las bases conceptuales de principios que rigen la Ciencia Ocupacional.

b) Asignaturas Generales:

Aportan los conocimientos del desarrollo del ser humano a través de los tiempos, haciendo énfasis en contenidos de cultura, estructura de grupos y las instituciones sociales. Dan al estudiante una visión humanística del hombre como objeto de estudio y trabajo.

c) Asignaturas Clínicas:

Aportan los conocimientos acerca de las patologías, pronóstico y terapéuticas de las enfermedades que afectan los distintos órganos y sistemas del cuerpo humano. Dan al estudiante contenidos que le permiten identificar las entidades más frecuentes y la discapacidad agregada a la lesión.

d) Asignaturas Profesionales: 
Aportan al estudiante contenidos y experiencias prácticas que le permiten analizar una actividad y sobre esta base, inferir que variables pueden interferir o favorecer los procesos terapéuticos. Para así, establecer los grados de funcionalidad, determinar objetivos y modelos de atención y prevención de la discapacidad.

Un núcleo de transversalidad, que permite recoger y unir todos los saberes dados en las asignaturas formativas, son las competencias comunicativas que adquiere el terapeuta. En este ámbito se alcanzan preferentemente conocimientos teóricos y técnicos (competencia pragmática, competencia estratégica).

\section{Desde los saberes integrales del Plan de estudio.}

\section{a) Actividades de Autocuidado.}

Se intenta destacar, en la formación del estudiante la importancia de la cultura del autocuidado responsable; el hombre de hoy requiere criterios sostenibles para encauzar adecuadamente desde sus necesidades más elementales, tales como la alimentación, la comunicación, la administración de recursos y la sexualidad, para mejorar la calidad de vida.

Se desarrolla un Programa de Mantenimiento Proactivo de la Salud, tomando como líneas maestras los distintos saberes de la humanidad que buscan una mejor calidad de vida y cuyo enfoque intenta sistematizar el conocimiento que reconozca al hombre como un ente holístico que simultáneamente relaciona cuerpo, mente, emociones y espíritu.

b) Actividades de Productividad.

Se propone que el estudiante sepa apoyar el desarrollo del máximo potencial de las personas en el ambiente que lo rodea, logrando así el desarrollo propio.

Al mismo tiempo, ayudar a quien lo solicite a rescatar su máxima productividad humana, esto es, su capacidad para generar riqueza y bienestar, entendido esto como alegría, entusiasmo y salud. Proporcionando para estos fines no solo la motivación, sino él "como" (Esto es: la tecnología necesaria para ello).

Que se logre practicar y difundir las nuevas tecnologías de desarrollo humano tales como: El Pensamiento Sistémico, la Integración Socio Comunitaria y todos aquellos aspectos que unen el pensamiento científico con las ciencias humanas. Contribuir así, al vínculo entre la ciencia (tecnología) y el bienestar integral del ser humano.

c) Actividades de Tiempo Libre.

Se espera que los estudiantes comprendan y valoren el deporte y las actividades de esparcimiento como elementos de desarrollo integral, factores protectores de la salud y de la Calidad de Vida; asimismo se espera que analicen y valoren el Tiempo Libre y la actividad de esparcimiento como un área de Desempeño Ocupacional y que manejen herramientas básicas para la evaluación y el diseño de actividades de Tiempo Libre en población sana y en Personas con Discapacidad.

Un núcleo de transversalidad, de los saberes integrados a través del plan de estudio, son las competencias comunicativas que adquiere el terapeuta ocupacional. En este ámbito se alcanzan preferentemente conocimientos acerca de la naturaleza social de ser humano y la creación de sistemas de valores (competencia social, competencia sociocultural y competencia intercultural). El manejo de la de una favorable interacción comunicativa, entrega a la intervención profesional un enfoque democrático, centrado en el respeto por el otro y la tolerancia.

\section{3.- Desde el interés del Licenciado en Ciencia de la Ocupación:}


Entre las consideraciones relacionadas con este punto, se debe establecer que en el horizonte práctico del ser humano, se distingue un actuar productivo cuyo fin es la obra ( ergon) ${ }^{*}$ donde el saber se ajusta con miras a la producción de determinados objetos para la satisfacción de necesidades explícitas. El sentido ocupacional de la tarea productiva se centra en la posibilidad que aporta esta actividad para alcanzar la completud de cada ser a su ser específico; que entrega la alternativa de "hacerse" de un modo no predeterminado por una naturaleza.

Toda acción tiene un deseo que la motiva; hablar, trabajar, pensar, amar son actos provocados por la necesidad de decir, hacer, entender y querer a alguien. Las necesidades son el motor de la vida. Es el motivo del movimiento, nada impulsa a la acción que no se apetece.

a) Actividades para la satisfacción de necesidades básicas:

Analizar y estudiar los bienes y servicios básicos que es ser humano contemporáneo demanda con el propósito de sobre vivir. La competencia inevitable y la cooperación como medio de obtención.

Se busca, identificar la vinculación entre desarrollo y necesidades humanas al mismo tiempo del logro del desarrollo y la autodependencia.

b) Actividades para el desarrollo de deseos individuales y/o grupales:

Analizar y estudiar los requerimientos del ser humano más allá de las necesidades básicas, los deseos individuales o colectivos locales que son específicos y cuyo proceso de satisfacción son ilimitados y diversos.

Se busca, colaborar en la identificación de ocupaciones e intercambio de ellas de acuerdo a las habilidades particulares.

c) Actividades con significado trascendente en el ser humano:

Analizar y estudiar los comportamientos deliberados del ser humano, orientados en sustituir un estado de menor satisfacción por uno de mayor satisfacción cada vez superior, de acuerdo a las emociones y valoraciones. Reconociendo el proceso que la gente piensa, siente, aprende, valora y actúa.

La actividad psíquica que acompaña a toda actividad humana es un vinculo comunicativo con otros, la interrelación de los sujetos nos presenta una situación comunicativa donde se manifiesta una situación activa por naturaleza. Todas las competencias comunicativas, descritas en el enfoque comunicativo, se transversalizar y se democratizan. La licenciatura en ocupación perfila un profesional hábil en los procesos de mediación, para contribuir al bienestar cotidiano del ser humano que interactúa con el mundo a través de la ocupación.

\section{4.- Desde el interés del Terapeuta Ocupacional}

a) Actividades para intervenir terapéuticamente en niños y adolescentes con discapacidad o en riesgo de adquirirla:

Aportan los conocimientos y experiencias para manejar:

- Modelos de prevención de la discapacidad física y psicosocial en niños y adolescentes.

- Modelos de rehabilitación física y psicosocial en niños y adolescentes. Modelos de integración socio cultural en niños y adolescentes.

b) Actividades para intervenir terapéuticamente en Adultos con discapacidad o en riesgo de adquirirla: 
Aportan los conocimientos y experiencias para manejar:

- Modelos de prevención de la discapacidad física y psicosocial en personas adultas.

- Modelos de rehabilitación física y psicosocial en personas adultas.

- Modelos de integración sociocultural en personas adultas.

c) Actividades para intervenir terapéuticamente en senescentes con discapacidad o en riesgo de adquirirla:

Aportan los conocimientos y experiencias para manejar:

- Modelos de prevención de la disfuncionalidad del adulto mayor.

- Modelos de integraciòn en redes asistenciales.

- Modelos para el fomento y permanencia de la autonomía.

Un núcleo de transversalidad, que permite recoger y unir todos los conocimientos contenidos en las asignaturas formativas de índole profesional, son las competencias comunicativas que desarrolla el terapeuta ocupacional. En este ámbito se alcanzan preferentemente conocimientos teóricos y técnicos (competencia pragmática, competencia estratégica), para abordar los principales problemas de salud.

(*) Ergon, ergón concepto raíz, utilizado en terapia ocupacional para designar el quehacer del ser humano basado en el desarrollo de las virtudes (competencias) para alcanzar un bienestar; ejemplo: ergoterapia

\section{" Conclusiones "}

La consideración del enfoque comunicativo, en cuanto componente de fundamento en modelos de enseñanza, abre paso a un proyecto de convivencia humana al interior de una práctica democrática y de una concepción de cultura comunicativa que aún está pendiente cuando se aplica en la dinámica didáctica.

Si la capacidad de los docentes queda definida exclusivamente por conocer, producir y transferir conocimientos, lo más probable es que - aún siendo loable cada uno de los aspectos anteriores - conduce a un excesivo tecnicismo disciplinar. Habría que preguntarse si insistir en esta fórmula responde a aquello que futuros profesionales requieren en una sociedad abierta a la pluralidad y el consenso. Un proceso de formación global demuestra las limitaciones de la tendencia tradicional de enseñanza al no permitir que el conocimiento o saber acumulado se prive de construir vínculos sociales y personales más profundos. Un camino, como lo ha presentado este artículo, es el del Enfoque Comunicativo.

Puntualmente, en el caso de formación de Terapeutas Ocupacionales, los 4 niveles formativos se integran en las competencias comunicacionales alcanzados en el curriculum, los que se representa en la siguiente tabla:

\begin{tabular}{|l|l|}
\hline \multicolumn{1}{|c|}{$\begin{array}{c}\text { COMPETENCIAS } \\
\text { COMUNICATIVAS }\end{array}$} & \multicolumn{1}{c|}{$\begin{array}{c}\text { COMPETENCIAS DEL } \\
\text { TERAPEUTA OCUPACIONAL }\end{array}$} \\
\hline Competencia linguística & Manejo lenguaje técnico y contextual \\
\hline Competencia paralingûistica & $\begin{array}{l}\text { Actitud que propicie la acción, impulse iniciativas, } \\
\text { comportamiento instigador. }\end{array}$ \\
\hline Competencia pragmática & Saber hacer técnico, manejo de métodos y procedimientos, \\
\hline Competencia discursiva & Saber decir, explicar, dialogar y escuchar \\
\hline \hline Competencia sociocultural & Evaluar, conocer e intervenir en los contextos ocupacionales \\
\hline
\end{tabular}




\begin{tabular}{|l|l|}
\hline & para condicionar factores físicos y ambientales. \\
\hline Competencia social & $\begin{array}{l}\text { Evaluar, conocer e intervenir en los componentes sociales del } \\
\text { contexto ocupacional }\end{array}$ \\
\hline Competencia estratégica & Actitud y habilidades para la mediación y la facilitación social. \\
\hline Competencia intercultural & $\begin{array}{l}\text { Actitud y habilidades para la Intermediación entre los diversos } \\
\text { grupos sociales, entre distintos estilos de cuidados de la salud, } \\
\text { entre variados modelos de intervención oficial y su aplicación } \\
\text { subcultural. }\end{array}$ \\
\hline
\end{tabular}

Desde este enfoque teórico (comunicacional) el Terapeuta Ocupacional, como profesional de la salud poseedor de competencias intervinculantes; se perfila para contribuir a la atención a la atención para las necesidades de diferentes grupos de personas. Ello, con acciones de mediación para distender caos, crisis, violencia social e intercultural. Desde una mirada de la integración de la compleja dinámica que presentan los estados de conflictividad de los seres humanos en el tiempo actual.

\section{- Referencias "}

(1) Austin, J. (1982, reed.): Cómo hacer cosas con palabras. Palabras y acciones. Barcelona. Paidós.

(2) Berutto, G. (1979): La sociolingüística. México. Nueva Imagen.

(3) Ducrot, O. et al (1996 reed.): Diccionario enciclopédico de las ciencias del lenguaje. Madrid, Siglo XXI

(4) Habermas, J. (1987): Teoría de la acción comunicativa. vol. I. Buenos Aires. Taurus.

(5) Llobera, M. et al (1995): Competencia comunicativa. Barcelona, EDELSA

(6) Marcos M. et al (1991): Lingüística Aplicada. Madrid. Síntesis.

(7) Parret, H. (1993): Semiótica y Pragmática. Buenos Aires. EDICIAL.

(8) Reyes, G. (1990): Lingüística pragmática. Barcelona. Montecinos.

(9) Ricoeur, P. (1976): Exégesis y hermenéutica. Madrid. Cristiandad.

(10) Searle, J. (1990, reed.): Actos de habla. Madrid. Cátedra.

(11) Lomas C. et al. El enfoque Comunicativo de la enseñanza de la lengua.(1994), Barcelona. Paidós. pág.23. 